\title{
Data-Driven Crowd Evacuation Simulation Method Based on Raspberry Pi
}

\author{
Chengming ZHANG, Baoxu AN, Yibo WANG and Dianjie LU ${ }^{1}$ \\ School of Information Science and Engineering, Shandong Normal University, China
}

\begin{abstract}
When public emergencies or disasters occurred, such as fires and earthquakes, safety accidents with group trampling can easily be caused. In the previous work, the study of crowd evacuation mainly adopted artificial parameters for simulations. To overcome these problems, we propose a data-driven crowd evacuation simulation method based on Raspberry Pi. First, we configure Raspberry Pi as sensor node, which is connected the wireless WiFi network card with monitoring function and the infrared sensor module with detection function. And then the sensor node was deployed to collect real data of crowd evacuation in real scenarios. Second, the curve fitting method is used to deal with the functional relationship between crowd density and average evacuation speed. In terms of crowd evacuation simulation calculation, we present Crowd Density-based Reciprocal Velocity Obstacle (CD-RVO) algorithm which drive individual speed changes with crowd density, to optimize the options for safety exit of individuals and reduce evacuation time effectively. Finally, the experimental results show that the proposed method can simulate the process of crowd evacuation realistically and improve crowd evacuation efficiency.
\end{abstract}

Keywords. Raspberry Pi, Crowd evacuation simulation, Real data, Crowd density, Data-driven.

\section{Introduction}

With the accelerating process of urbanization, the number of crowded public places [1-2] is increasing. Due to the concentrated distribution and complicated layout of these public plazas, it is easy to occur safety accidents. Therefore, how to evacuate people safely and efficiently in public places has become a hot issue. In real scenarios, most of the crowd evacuation processes are simulated through on-site exercises to reduce the frequency of safety accidents occurrence. With the rapidly development technology of information, the computer technology provides a strong support for improving crowd evacuation simulation [3-4] efficiency. Most methods of crowd evacuation simulation mainly focus on obstacle avoidance and path planning mechanism. Typical crowd evacuation models include fluid mechanics [5-6] models, method models based on individual rules [7-8] and crowd simulation models based on social forces [9-11]. Most of the experimental data in the above models or methods are based on manmade assumptions, reducing the realism of crowd evacuation simulation in emergencies. Therefore, it is of great significance to obtain the real data of crowd movement and

${ }^{1}$ Corresponding Author, Dianjie LU, School of Information Science and Engineering, Shandong Normal University, China; E-mail: ludianjie@sdnu.edu.cn. 
apply it to the crowd evacuation simulation. In recent years, the exploration of real data [12-14] to simulate crowd evacuation [15-16] has also attracted attention of researchers. Nevertheless, it is hard to extract crowd movement trajectory and filter complicated crowd data. It is a hot topic that how to obtain crowd movement data efficiently in real scenarios and apply it to crowd evacuation simulation, improving the realism of crowd evacuation simulation.

In order to reproduce the specific process of crowd evacuation more realistically and overcome the disadvantages of complexity data process, a data-driven crowd evacuation simulation method based on Raspberry $\mathrm{Pi}$ is proposed in this article. Our key contributions are summarized as follows:

- A novel mechanism for collecting real data is proposed. In this mechanism, we reset Raspberry Pi which connect the wireless WiFi network card with monitoring function and the infrared sensor module with detection function, to collect real data of crowd evacuation in scenarios.

- We construct an exponential function to analyze the influence of crowd density on average evacuation speed during the process of crowd movement. A curve fitting method is applied to crowd dataset which collected by Raspberry $\mathrm{Pi}$ in real scenarios. We build an exponential function to fit the relationship between crowd density and average speed of individuals, and apply it to avoidance strategies for crowd evacuation simulation.

- In terms of crowd evacuation calculation, we proposed a Crowd Density-based Reciprocal Velocity Obstacle (CD-RVO) algorithm which merge exponential function of crowd density and average speed, to optimize the options of safe exit for individual and reduce time of crowd evacuation. Additionally, we establish a crowd simulation system based on real data to visualize the simulation results.

The remainder of this paper is organized as follows. Section 2 is contributed to review of related studies on crowd simulation. Then, we present a method for collecting real data of crowd evacuation in Section 3 and a CD-RVO algorithm is introduced in Section 4. Next, Section 5 reports the simulation results, and our conclusions are presented in Section 6.

\section{Related Work}

\subsection{Data-Driven Method}

In order to simulate crowd evacuation more realistically in real scenarios, many scholars have adapted available methods including GPS positioning [14], Bluetooth positioning [17] and video extraction [18-19] to track movement trajectory and collect real data for crowd evacuation simulation researches. Xu et al. [20] used the GPS device of mobile terminal to collect information of individual location and extract crowd data. Due to the imprecision of positioning methods of Bluetooth and GPS, researchers impossible record the crowd data accuracy in multi-layer buildings indoor. The method mentioned above is difficult to apply to crowd evacuation simulations in multi-layer buildings scenarios. Kim et al. [21] proposed an adaptive data-driven algorithm for interactive crowd simulation. Lerner et al. [22] presented an 
example-based crowd simulation method. Liu et al. [23] proposed a video data-driven social force model for simulating crowd evacuation. However, the above video capture method has some limitations. The crowd trajectories extraction is influenced by factors such as visibility of environment, crowd density and video angle of visual filed. Therefore, we propose a method for collecting real data of crowd evacuation based on Raspberry Pi, to collect and process the real data for crowd evacuation simulation.

\subsection{Crowd Evacuation Method}

In crowd evacuation simulation model, both global path planning and local collision avoidance are considered for the processing of crowd movement from one position to next target. Wu et al. [24] proposed a real-time emotion-integrated path planning algorithm to explore the global path. Liu et al. [25] combined an Artificial Bee Colony algorithm with Particle Swarm Optimization algorithm for the path planning of crowd evacuation simulation. Zhang et al. [26] proposed an improved social force model to simulate and reproduce the process of group gathering. Berg et al. [27] put forward a Reciprocal Velocity Obstacle (RVO) algorithm which considers the motion speed of its neighbor and the last moment speed of individual comprehensively. However, the traditional RVO model assumes that movement of crowd keep a constant speed during the entire evacuation process unless the avoidance strategy is triggered. Therefore, we explore the relationship between crowd density and average evacuation speed, taking real dataset of crowd evacuation collected by Raspberry Pi.

\section{Data-Collection Based on Raspberry Pi}

Our goal is to drive the crowd evacuation more realistically, we propose a method of data-driven crowd evacuation simulation method based on Raspberry Pi.

\subsection{Initialization Raspberry Pi system}

As a device of data collection and infrared sensing function, Raspberry $\mathrm{Pi}$ is equipment with operation environment, software system and compile program. Firstly, we use Linux command internally for the Raspberry Pi to configure the internal software environment and system. Secondly, the Raspberry Pi connected with the wireless WiFi network card and the infrared sensor module, is deployed as sensor node in real scenarios. We can get the location of individual within the coverage of wireless network. Finally, the terminal server controls command and receives real data of crowd evacuation collected by sensor nodes. These real data are applied to crowd evacuation simulation, which include the number of individual, average speed and crowd density.

\subsection{Crowd Data Collection in Real Scenarios}

\subsubsection{Collection Crowd Density}

Driven by the tremendous popularity by smartphones, the number of mobile internet users has reached 986 million by December 2020 in China. Without loss of generality, we consider a special scenario where everyone has one mobile phone. The method of 
data-collection based on Raspberry Pi identify the number of smartphone which send wireless signal, to count the number of people in real scenarios. Sensor node monitors the wireless signals of crowd mobile phone and captures data packets in the scenarios. According to the number of MAC addresses of mobile phone from the data packets, we get the number of people $\mathrm{N}$ in real scenarios. Generally, the wireless signal coverage area $\mathrm{S}$ of the Raspberry $\mathrm{Pi}$ with monitoring function ranges between 52 square meters to 60 square meters. As the crowd area $\mathrm{S}$ and the number of people $\mathrm{N}$ are obtained, the crowd density is $\rho=\mathrm{N} / \mathrm{S}$. And then, the result of crowd density is storage in terminal server.

\subsubsection{Average Speed Collection}

We collect the time information of crowd when the sensor node detect individual across the coverage area. We adopt the method which match the time of MAC address from individual mobile phone to the time of infrared detection, to assure the passing time of individual. Generally, we deploy sensor node A and sensor node B respectively in real scenarios. The passing time, $\mathrm{T} 1$, represents the time of individual detected by sensor node A with infrared detection function in room. We set the room as dangerous area in real scenarios. Likewise, T2 is the passing time which detected by sensor node B with infrared detection function when individual arrive at safe area. We use the wireless WiFi network card of sensor node with monitoring function to capture the detected time of individual carrying the mobile phone in monitored area. The detected time Tw1 and Tw2 indicate that the individual is detected by sensor node at last time in the room and the first time in safe area respectively. If Tw1 matches T1 and Tw2 matches T2 respectively, we confirm that the passing time sensing by sensor node with infrared detection function is accurate. Then, the evacuation time of crowd is $|\mathrm{T}|=\mathrm{T} 2-\mathrm{T} 1$, which represents individuals arrive at the safe area from the room. We obtain the average speed $\mathrm{V}$ of individual, $\mathrm{V}=\mathrm{L} / \mathrm{T}$, where $\mathrm{L}$ is the evacuation distance of crowd in the scenarios. The specific schematic of time collection for crowd evacuation based on Raspberry Pi is shown in figure 1.

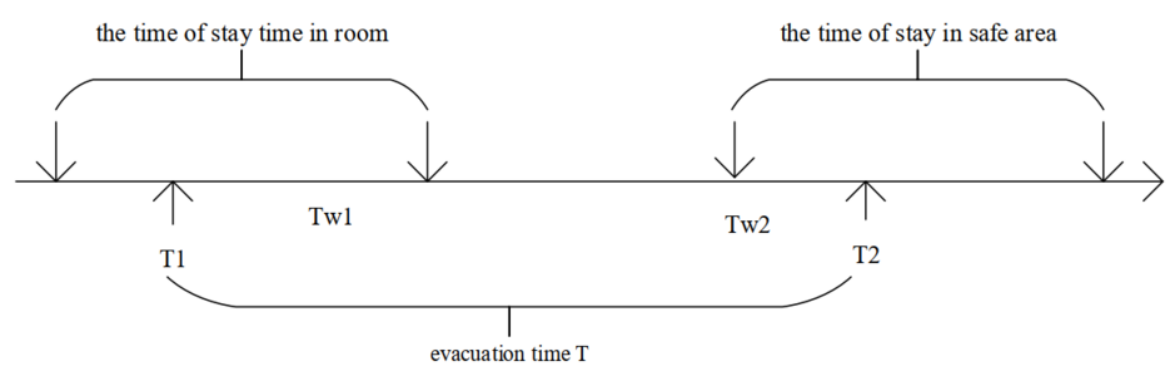

Figure 1. Time collection for crowd evacuation.

\subsubsection{The Fitting Curve for Crowd Density and Averaged Speed}

The method of data-collection based on Raspberry Pi collect real dataset, including evacuation distance, crowd density and evacuation time in real scenarios. In order to explored the relationship between crowd density and average speed of individual, we make a curve graph using real data of crowd evacuation collected by Raspberry Pi. The exponential function is used to fit the curve with the original data. And it is allowed not to pass through all data points in this process. We obtain the result of $\mathrm{R}$-square $=0.9816$, 
which is close to 1.0. The result proves that the process for curve fitting is better. We build an exponential function (equation (1)) for the relationship between crowd density and average speed of individuals. In figure 2, it is clear that the average speed of individuals decreases as the crowd density increases. Most importantly, the feature of the relationship between crowd density and average speed is applied to crowd evacuation simulation.

We express the exponential function between crowd density and average speed as

$$
V=0.9652 * \rho^{-0.5694}
$$

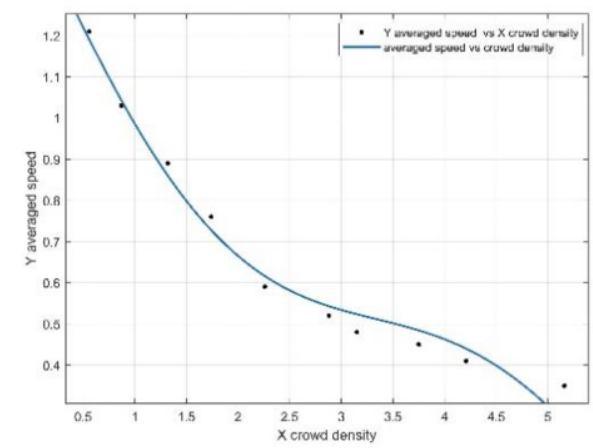

Figure 2. The fitting curve of crowd density and average speed fitting curve.

\section{Crowd Density-based Reciprocal Velocity Obstacle}

In order to optimize the evacuation path, we consider the effect of crowding on evacuation speed. A Crowd Density-based Reciprocal Velocity Obstacle (CD-RVO) algorithm are proposed to simulate crowd evacuation more realistically. Firstly, the speed of individual movement at time $t$ can be obtained by calculating equation (1). Secondly, we estimate evacuation time of individual arrive at safety area, to optimize the options of safe exit for individual and calculate preferred velocity of each individual. Finally, we update individual velocity for the next moment. Crowd density drives velocity change of individuals, so as to affect the whole trend of crowd changes.

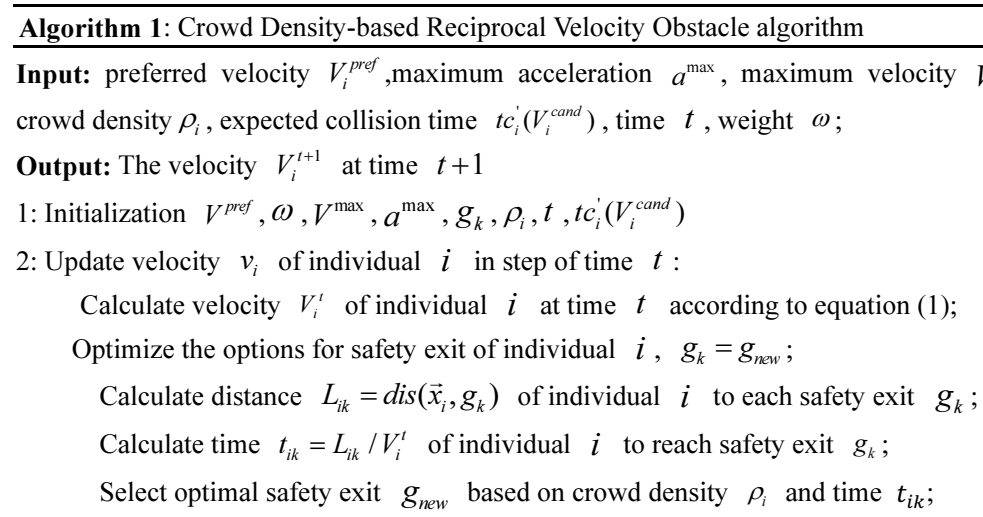




\author{
Update the preferred speed $v_{i}^{\text {pref }}$; \\ 3: Calculate candidate velocity $V^{\text {cand }}\left(V_{i}\right)$; \\ 4: Calculate velocity $V_{i}^{t+1}$; \\ 5: Repeat step 2 to step 4 until individual $i$ arrive at safety exit.
}

\title{
5. Experiment Analysis
}

In this section, we evaluate the proposed method in three parts, and initialize individual with real data collected by Raspberry Pi. The first part compares the performance of traditional RVO and CD-RVO algorithm and the second part visualize the crowd evacuation simulation process.

\subsection{Comparison of $R V O$ and $C D-R V O$}

In this experiment, we explore the realism of traditional RVO and CD-RVO algorithm for crowd simulation and compare evacuation time of two algorithms. We drown on the scenario in the hall of teaching building which has two exits. In figure 3, we can see that the traditional RVO algorithm is prone to congestion at the same safe exit during the process of crowd evacuation. Differently, the CD-RVO algorithm proposed takes into account the factor of crowd density on the average speed of individuals. Thus, individuals choose different safe exits to move quickly in the process of crowd evacuation, which alleviate the pressure of safe exits effectively and avoid crowding to one exit.

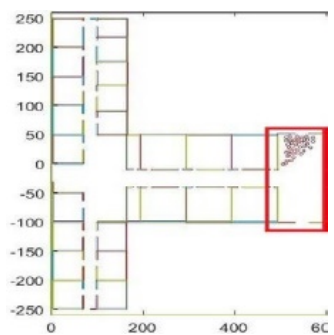

(a)

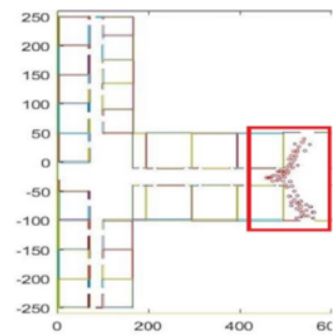

(b)

Figure 3. Comparison of simulation process for RVO and CD-RVO . (a) The simulation process of RVO. (b) The simulation process of CD-RVO.

\subsection{Visualization of the Crowd Evacuation Simulation Process}

To verify the proposed method can enhance the realism of crowd evacuation simulation, we implement a crowd simulation system based on real data to visualize the simulation results. We set an example with 400 individuals and two safety exits in the hall of teaching building scenario. The evacuation process of whole scenario is shown in figure 4 (a) which is a vision graph of the hall in teaching building scenario at distance view. figure 4 (b) is a is a closed view of the process for crowd evacuation simulation process in the hall of teaching building scenario. 

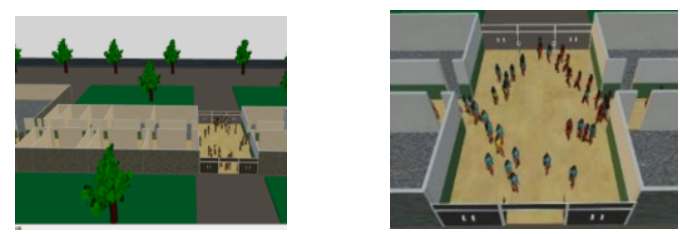

Figure 4. Simulation results of crowd evacuation in hall of teaching buildings scenario. (a)Vision View. (b) Closed view.

\section{Conclusion and Future Work}

We propose a data-driven crowd evacuation simulation method based on Raspberry Pi. First, the Raspberry Pi connected the wireless WiFi network card and the infrared sensor module, are deployed as sensor node to collect real data of crowd movement in scenarios. Second, we present curve fitting method which is used to deal with the functional relationship between crowd density and average speed of individuals. Then, a CD-RVO algorithm proposed to optimize the options of safe exit for individual. Finally, we implement a crowd simulation system based on real data collected by Raspberry Pi to visualize the results of our theoretical analysis. The proposed method can more realistically simulate crowd evacuation and can provide a reference for crowd evacuation navigation.

\section{Acknowledgments}

This work was supported in part by the National Natural Science Foundation of China under Grants 61972237, in part by the Joint funds for Smart Computing of the Natural Science Foundation of Shandong Province under Grant No. ZR2020LZH003, in part by the Shandong Provincial Natural Science Foundation of China under Grant No. ZR2019MF017.

\section{References}

[1] J. Ma, W. Song, S. Lo, et al., "New insights into turbulent pedestrian movement pattern in crowd-quakes," Journal of Statistical Mechanics Theory and Experiment, vol. 2013, no. 2, pp. 583-602, 2013.

[2] R. Shang, P. Zhang, M. Zhong, "Investigation and analysis on evacuation behavior of large scale population in campus," Procedia Engineering, vol. 52, no. 7, pp. 302-308, 2013.

[3] F. Qiu, X. Hu, "Modeling group structures in pedestrian crowd simulation," Simulation Modelling Practice and Theory, vol.18, no. 2, pp. 190-205, 2010.

[4] X. Zhao, Y. Zhang, D. Kong, et, al., "Field-based crowd simulation," Journal of Image and Graphics, vol. 18, no. 3, pp. 344-350, 2013.

[5] R. Narain, A. Golas, S. Curtis, et al., "Aggregate dynamics for dense crowd simulation," ACM Transactions on Graphics, vol. 28, no. 5, pp. 1-8, 2009.

[6] H. Jiang, W. Xu, T. Mao, et al., "Continuum crowd simulation in complex environments," Computers and Graphics, vol. 34, no. 5, pp. 537-544, 2010.

[7] P. Tissera, A. Printista, M. Errecalde, "Evacuation simulations using cellular automata," Journal of Computer Science and Technology, vol. 7, pp. 14-20, 2007.

[8] M. Xiong, M. Lees, W. Cai, S. Zhou, et al., "Analysis of an efficient rule-based motion planning system for simulating human crowds," The Visual Computer, vol. 26, no. 5, pp. 367-383, 2010. 
[9] L. Hou, J. Liu, X. Pan, et al., “A social force evacuation model with the leadership effect," Physica A Statistical Mechanics and Its Applications, vol. 400, no. 2, pp. 93-99, 2014.

[10] P. Saboia, S. Goldenstein, "Crowd simulation: applying mobile grids to the social force model," Visual Computer, vol. 28, no. 10, pp. 1039-1048, 2012.

[11] Y. Qu, Y. Dan, "Modeling the evacuation behavior considering the effect of dangerous source," Procedia - Social and Behavioral Sciences, vol. 138, pp. 800-810, 2014.

[12] A. Bera, S. Kim, D. Manocha. "Modeling trajectory-level behaviors using time varying pedestrian movement dynamics," Collective Dynamics, vol. 3, pp. 1-23, 2018.

[13] M. Cao, G. Zhang, M. Wang, et al., "A method of emotion contagion for crowd evacuation," Physica A: Statistical Mechanics and Its Applications, vol. 483, pp. 250-258, 2017.

[14] X. Song, Q. Zhang, Y. Sekimoto, et al., "Modeling and probabilistic reasoning of population evacuation during large-scale disaster," Proc. of 19th Sigkdd Conference on Knowledge Discovery and Data Mining, 2013, pp. 1231-1239.

[15] P. Torrens, X. Li, W. Griffin, "Building agent-based walking models by machine-learning on diverse databases of space-time trajectory samples," Transactions in GIS, vol. 15, pp. 67-94, 2011.

[16] E. Ju, M. Choi, M. Park, J. Lee, et al., "Morphable crowds," ACM Transactions on Graphics, vol. 29, no. 6 , pp. 1-10, 2010 .

[17] L. Schauer, M. Werner, “Analyzing pedestrian flows based on WiFi and Bluetooth captures,” Digital Marketplaces Unleashed, 2015.

[18] M. Zhao, S. Turner, W. Cai, "A data-driven crowd simulation model based on clustering and classification," Proceedings of the 2013 IEEE/ACM 17th International Symposium on Distributed Simulation and Real Time Applications, 2013, pp. 125-134.

[19] Z. Yao, G. Zhang, D. Lu, H. Liu, "Data-driven crowd evacuation: A reinforcement learning method," Neurocomputing, vol. 366, pp. 314-327, 2019.

[20] X. Xu, L. Zhang, S. Sotiriadis, et al., "CLOTHO: a large-scale internet of things based crowd evacuation planning system for disaster management," IEEE Internet of Things Journal, vol. 5, no. 5, pp. 3559-3568, 2018.

[21] S. Kim, A. Bera, A. Best, R. Chabra, et al., "Interactive and adaptive data-driven crowd simulation," Proceedings of the 2016 IEEE Virtual Reality, 2016, pp. 29-38.

[22] A. Lerner, Y. Chrysanthou, D. Lischinski, "Crowds by Example," Computer Graphics Forum, 2007, pp.655-664.

[23] B. Liu, H. Liu, H. Zhang, X. Qin, "A social force evacuation model driven by video data," Simulation Modelling Practice and Theory, vol. 84, pp. 190-203, 2018.

[24] Y. Wu, Y. Du, Y. Ye, "Real-time emotion-integrated crowd path planning," Journal Of Image and Graphics, vol. 20, no. 9, pp. 1262-1274, 2015.

[25] H. Liu, Y. Sun Y, Y. Li, "Modeling and path generation approaches for crowd simulation based on computational intelligence," Chinese Journal of Electronics, vol. 21, no. 4, pp. 636-641, 2012.

[26] H. Zhang, H. Liu, X. Qin, B. Liu, "Modified two-layer social force model for emergency earthquake evacuation," Physica A: Statistical Mechanics and Its Applications, vol. 492, pp. 1107-1119, 2018.

[27] J. Berg, S. Patil, J. Sewall, et al., "Interactive navigation of individual agents in crowded environments," Symposium on Interactive 3D Graphics and Games. 2008, pp. 139-147. 